International Journal of Computer Networks \& Communications (IJCNC) Vol.3, No.1, January 2011

\title{
AN EfFicient Cluster BASEd APPROACH FOR Multi-Source Multicast Routing Protocol IN MOBILE AD HOC NETWORKS
}

\author{
R. Pandi Selvam ${ }^{1}$ and V.Palanisamy ${ }^{2}$ \\ ${ }^{1,2}$ Department of Computer Science and Engineering \\ Alagappa University, Karaikudi, Tamil Nadu, India \\ pandi0703@yahoo.com vpazhanisamy@yahoo.co.in
}

\begin{abstract}
Mobile ad hoc networks are becoming an important concept of modern communication technologies and services. It provides some advantages to this communication world such as self-organizing and decentralization. In this paper, we are going to design a cluster-based multi source multicast routing protocol with new cluster head election, path construction and maintenance techniques. The main objective of this work to compute the maximum performance of proposed routing protocol in various environments, and also it has been compared with Multicast Ad-hoc On-Demand Distance Vector $(M A O D V)$ and On-Demand Multicast Routing Protocol (ODMRP) to prove the performance of delivery ratio, control overhead and forwarding efficiency.
\end{abstract}

\section{KEYWORDS}

Mobile Ad hoc Networks, Routing Protocols, MAODV, ODMRP, Multicasting, Clustering, Network Simulator

\section{INTRODUCTION}

Mobile ad-hoc networks (MANETs) also called ad hoc network which has collection of wireless devices without any stand-alone infrastructure and centralized manner. Moreover each node behaving with self-organizing and self-configuring feature i.e. acts as a router. With the above ethics this networks are particularly important and useful in emergency regions, such as battlefields and disaster areas [9]. Routing is an essential component of communication networks, which defines act of moving information from source node(s) to destination node(s) in an inter network. Multicast is a type of routing scheme, which transmit datagram's to a set of desirable destinations identified by a single address [6]. This routing scheme may be one-tomany or many-to-many. If the messages are communicated from one source to multiple destination address called one-to-many multicast routing. If it happens among the various sources to various destinations called many-to-many multicast routing scheme. There are many applications and services are achieved by multicast routing such as video conferencing, distance learning and video on-demand, etc. Based on the topology the existed ad hoc multicast routing protocols are classified into two categories i.tree based ii.mesh based. The tree based routing scheme has only one path between the source to receiver. MAODV, AMRIS, AMRoute are the best examples for the tree based scheme. But, the mesh-based routing scheme has multiple redundant paths between the sources to receivers. ODMRP, CAMP is the typical examples of mesh-based scheme [2]. Both are giving some salient features to mobile ad hoc network. In earlier days multicast routing protocols are designed with the multiple unicast system [9]. After that it had extended as multicast system, which has only one source in a multicast group. Recently, some prominent researchers have been proposed the cluster based multicast routing 
scheme for ad hoc networks [12] [13]. By this admissible work we can share different kinds of services or applications simultaneously in the multicast group. The reminder of this paper has four sections. The Section 2 has the related work. Section 3 explains the proposed cluster-based multi-source multicast routing protocol with new cluster head election, route construction and route maintenance procedure. Section 4 contains the NS2 implementation environment for the proposed clustering algorithm. Section 5 deals with results and discussion and conclusion described in Section 6.

\section{RELATED WORK}

The literature survey has been done to analyze the performance of two on-demand multicast routing protocols with the proposed routing protocol in mobile ad hoc network. Already a number of eminent researchers carried out some experiments to study the performance of multicast routing protocols such as MAODV, AMRoute, CAMP and ODMRP in mobile ad hoc environments and the [8] [10] references are explaining the survey of clustering algorithms. However, in the literature very few attempts are proved to evaluate the cluster- based multisource multicast routing protocol. In this work, we proposed to study the performance of two on-demand multicast routing protocols namely MAODV and ODMRP with the proposed CBMRP.

\subsection{The Multicast operation of Ad-hoc On-demand Distance Vector (MAODV)}

The Multicast operation of Ad-hoc On-demand Distance Vector (MAODV) is a reactive treebased multicast routing protocol [2]. It is an extension of the unicast routing protocol Ad-hoc On-demand Distance Vector (AODV). Like unicast routing operations, the every MAODV multicast group also has a sequence number to indicate the freshness of the routing information. In every group only one leader is elected with GROUP HELLO messages throughout the MANET to maintain the sequence number [1]. If, the group leader is by default the first node joining the group, but could also be another node when the first node leaves the group. In the MANET multicast transmission, every tree has to form with the on-demand manner to include all the group members and some non-members which are relay nodes [8]. The process of building such a tree is similar to the route discovery procedure in unicast routing: every time when a node wants to join a multicast group or to send a data packet to a multicast destination. This MAODV routing protocol works with RREQ message and RREP message. The RREQ broadcast throughout the network and send back with the RREP message. The nodes forwarding RREQ and RREP record the path backwards to the source of packet, as they will do in unicast routing. In this multicast tree link breakage is possible due to node movement and that topology. At that time of link breakage the group leader initiates local repair message to the node. Again, it broadcasts a RREQ message and waits for RREP from the group leader. By this means the tree is reconstructed to accommodate the topological change.

\subsection{The On-Demand Multicast Routing Protocol (ODMRP)}

The On-Demand Multicast Routing Protocol (ODMRP) is a soft state reactive mesh based multicast routing protocol. In this approach the source establishes and maintains group membership and multicast mesh on demand if it needs to send data packets to the multicast group, which is somewhat similar to MAODV [1] [4]. But, it builds a mesh instead of tree for packet transmission. A set of nodes, which is called forwarding group, participate in forwarding data packets among group members. When a source node needs the route to a multicast group, it begins to periodically broadcast a JOIN REQUEST message, which is forwarded by all the nodes in the MANET. When a group member receives such a message, it records the IP address of the node upstream to be 
the next hop for the source, and broadcasts a JOIN TABLE to its neighbor. On receipt of the JOIN TABLE, the neighbor node examines the table to see if it is the next hop for the source in one entry. If the answer is positive, the node sets itself to be a forwarding node and broadcasts its own JOIN TABLE to its neighbors as well. Thus the JOIN TABLE is sent back until is reaches the source [12]. At that time the forwarding group is formed and the route is built. From then on the data packets can be delivered to the receivers properly.

\section{Cluster-Based Multi-Source Multicast Routing Protocol}

Clustering is a familiar technique for grouping nodes that are close to each other in the network which prevents the flood of unnecessary packets and avoids wasting network bandwidth. In this technique, a large network can be divided into several sub-networks with only a few cluster heads maintains the local information with few packet losses [11]. The reference [2] [10] has various clustering algorithm for mobile ad hoc network. This clustering techniques are working with three important aspects which shown in Figure 1.

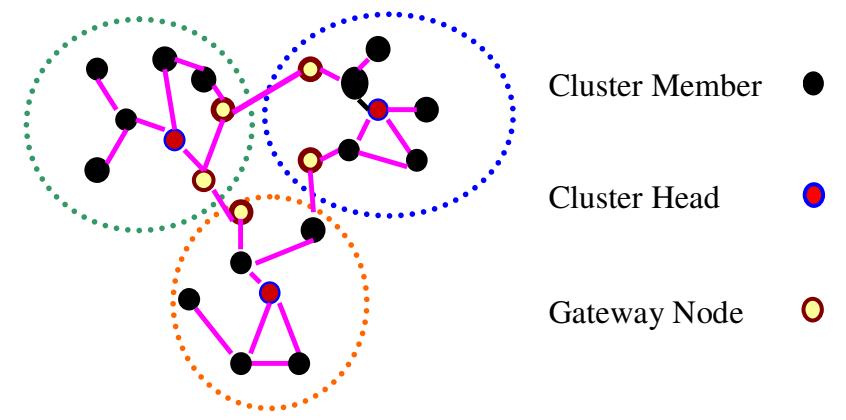

Figure 1. An Example of Clustered Network

Cluster Head $(\mathrm{CH})$ plays with superior power in the intra-cluster network which elected by the other member nodes. In our self-organized clustering scheme the cluster head only serves the purpose of providing a unique Integer Identification Number (ID) for the cluster, limiting the cluster boundaries. The section 3.1 explains the weight based clustering algorithm. Cluster Gateway (CG) is a non cluster-head node which is used to convey the routing information from one cluster to another. Cluster Member (CM) is a node that is neither a cluster head nor a cluster gateway [7]. The aim of the clustering algorithm is to elect some appropriate node as cluster heads and other member nodes are dominated by the $\mathrm{CH}$. The $\mathrm{CH}$ nodes are maintaining the cluster information which contains necessary information of clustering algorithm. After collecting the cluster information the node can exchange the cluster information with its neighbors which is used to construct the multicast path and that maintenance [5] [9]. The planned routing protocol developing procedures are explained below.

\subsection{Cluster Head Election}

Cluster head election is the complicated process of ad hoc network due to that dynamic topology [3]. We consider the every cluster has been formed by a graph represented $G=(V, E)$ where ' $V$ ' is a set of mobile nodes and ' $\mathrm{E}$ ' is the bi-directional link between the nodes. 


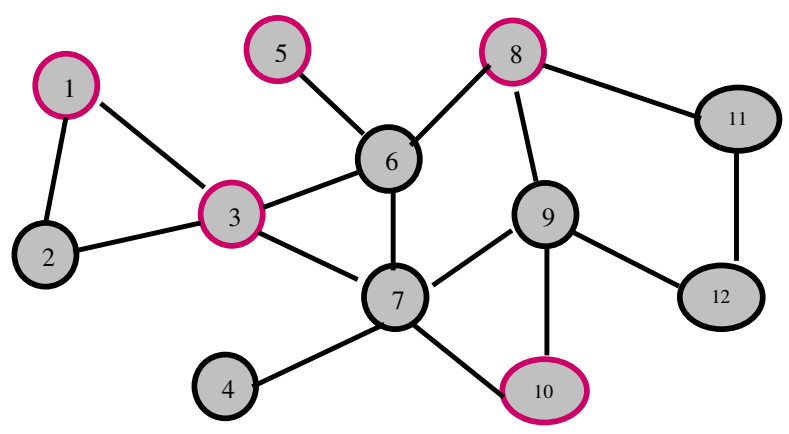

Figure 2. An Example of Weight calculation

For this work we have chosen the weight based clustering algorithm to elect the cluster head and path construction which defines the weight function $w(p)$ to calculate the weight of each node. For each node 'p' and the weight function $w(p)$ calculated as follows.

Where,

$$
w(p)=x \times a(p)+y \times b(p)+z \times c(p)
$$

a(p) number of multicast member nodes in one-hop $b(p)$ number of multicast member nodes in two-hop $\mathrm{c}(\mathrm{p})$ number of multicast member and cluster member nodes within two-hops.

In our clustering algorithm, the maximum hop distance taken from the ' $\mathrm{p}$ ' is two hops, and we ensure that each non cluster head node is managed by only one cluster head which is one of its neighbors within two hops. According that, we assign the variables $\mathrm{x}, \mathrm{y}, \mathrm{z}$, as constant. So, $\mathrm{x}=$ $3, y=2$ and $z=1$. The variable $x, y$ and $z$ are depends the number of hops.

$$
\begin{aligned}
& \mathrm{w}(1)=3 \times 1+2 \times 0+1 \times 4=7 \\
& \mathrm{w}(2)=3 \times 2+2 \times 0+1 \times 4=10 \\
& \mathrm{w}(3)=3 \times 1+2 \times 3+1 \times 9=18 \\
& \mathrm{w}(4)=3 \times 0+2 \times 2+1 \times 5=9 \\
& \mathrm{w}(5)=3 \times 0+2 \times 2+1 \times 4=8 \\
& \mathrm{w}(6)=3 \times 3+2 \times 2+1 \times 10=23
\end{aligned}
$$

$$
\begin{aligned}
& \mathrm{w}(7)=3 \times 2+2 \times 3+1 \times 10=22 \\
& \mathrm{w}(8)=3 \times 0+2 \times 3+1 \times 8=14 \\
& \mathrm{w}(9)=3 \times 2+2 \times 1+1 \times 8=16 \\
& \mathrm{w}(10)=3 \times 0+2 \times 2+1 \times 7=11 \\
& \mathrm{w}(11)=3 \times 1+2 \times 0+1 \times 4=7 \\
& \mathrm{w}(12)=3 \times 0+2 \times 2+1 \times 5=9
\end{aligned}
$$

From this calculation, the node 6 has the largest weight and it becomes the cluster head and other nodes are behaving as cluster member node.

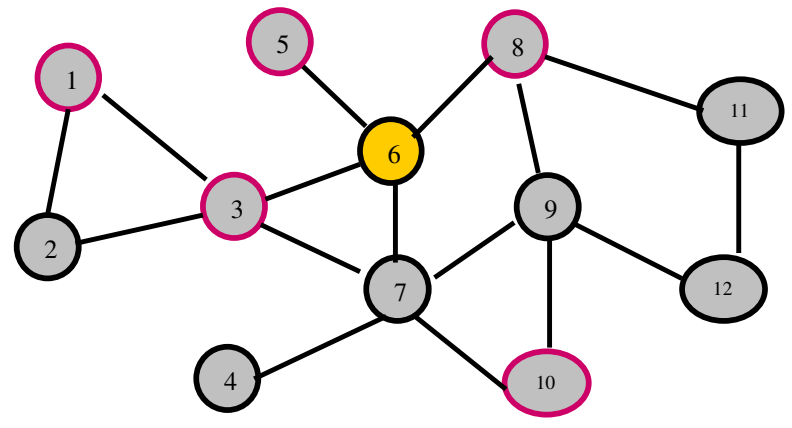

Figure 3. An Example of Cluster Head Election 
After the $\mathrm{CH}$ election, the network is ready to construct the multicast route and set forwarding clusters with cluster information which maintained by each node and the cluster member information maintained by cluster heads.

\subsection{Route Construction}

A routing protocol designs can be divided into two important phases, namely, route construction and route maintenance. During, the route construction phase, routes are connected between all pairs of nodes. The route maintenance phase takes care of maintaining the route due to the topological changes [9]. By setting proper clusters as forwarding clusters, data packets can be delivered along the routes connected by forwarding clusters. In a clustered network, the communication of nodes can be classified into two types. i.e. intra-cluster routing and intercluster routing. Intra-cluster routing is responsible for the communication of nodes within the same cluster. Since the cluster head maintains the cluster member information as well as local topology, within the same cluster. The inter-cluster routing explains receiver and the source between the different clusters. In this situation, the cluster head of the source sends a query to neighboring clusters through corresponding gateway nodes. The Figure. 4 and 5 explains the route construction by the first and all sources respectively. In cluster based routing protocol the Route Request Message (RREQ_MSG) will always follow a route by the following method:

$$
\text { Source } \rightarrow \text { Cluster Head } \rightarrow \text { Gateway } \rightarrow \text { Cluster Head } \rightarrow \text { Gateway } \rightarrow \cdots \rightarrow \text { Destination }
$$

Following the above cluster based broadcasting approach; the node S1 broadcasts RREQ_MSG to the entire network shows in Figure.1. It explains the message is only forwarded by necessary nodes and gateway nodes of each cluster. If this message is received by a cluster for the first time, the ID of the cluster is attached in the message before forwarding to its neighboring clusters. After receiving RREQ_MSG the multicast receivers then send Route Reply Message (RREP_MSG) along the same route. Once the RREP_MSG reaches the cluster source S1, the route connects the multicast source with multicast receivers is constructed. The above procedure only constructs the route connects the first source node $\mathrm{S} 1$ with that multicast receivers.
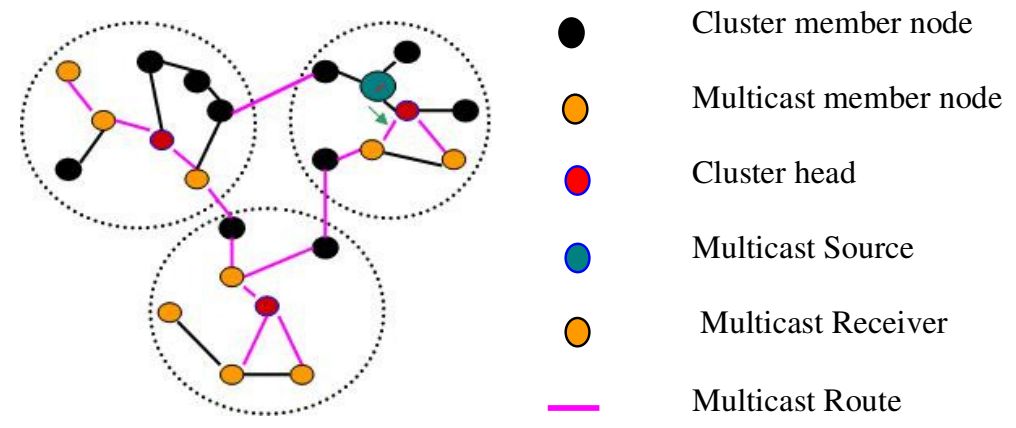

Figure 4. Route Constructed by the First Source

After that the new source S2 construct the route and connect it receivers, shown in Figure.5. The new source S2 is already in a forwarding cluster is able to send the message to neighboring cluster source and it can send the data packets to the receiver through the constructed multicast routes connected by forwarding clusters. 


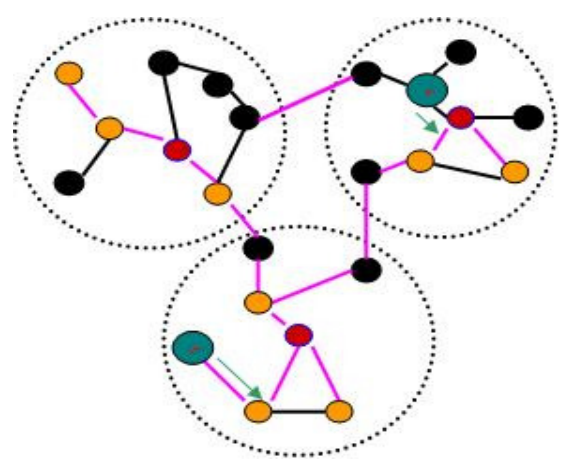

Figure 5. Route Constructed by all Sources

\subsection{Route Maintenance}

Due to the dynamic topology the route maintenance has a vital role in the multi source multicast environment. In this segment each node maintains the cluster member information with 1-hop neighbor. If any one node moves away from its cluster more than 2-hop, that node cannot be a member of the original cluster. By this way a node is able to maintain the local topology changes and sends an update message to its cluster head. The cluster head learns the intra cluster link states by updating cluster information. If there is occur any link breakage within the cluster can be easily solvable by asking the cluster head for an alternate route to the multicast member, as shown in Figure.6.In such cases some multicast members may not be connected to the multicast path due to the huge topology and cluster changes or after a long time. In these situations, we should reconstruct the multicast path at the Refresh Interval (RI) to ensure the system. At that time of reconstruction the source node construct the multicast path, along with Route Reconstruction (ROU_RECON) packet to the entire network.

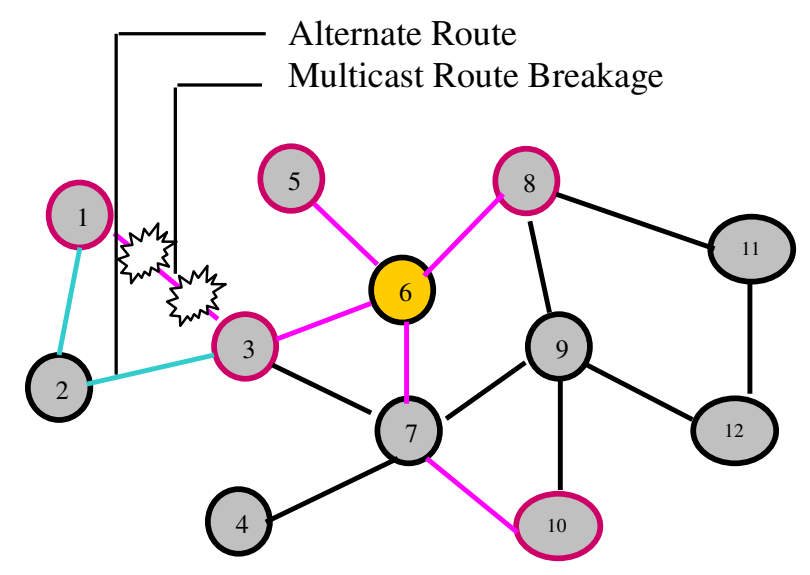

Figure 6. Route Maintenance in intra-cluster

\section{IMPLEMENTATION}

This proposed routing protocol has been implemented by the Network Simulator (NS2). The NS2 is mainly used to implement the routing protocols in the networking research. The [14] [15] references are mainly used for this implementation. We run several simulations under Linux, using the NS2 version ns-allinone-2.26. The simulation environment is composed of: 
Table 1. Simulation Environment

\begin{tabular}{|l|l|}
\hline Area & $1000 * 1000 \mathrm{~m}$ \\
\hline Number of nodes & 200 \\
\hline Transmission Range & $500 \mathrm{~m}$ \\
\hline Number of Senders & $1,5,10,15,20$ \\
\hline Number of Receivers & $10,20,30,40,50$ \\
\hline Simulation duration & $500 \mathrm{sec}$ \\
\hline Packet Size & $512 \mathrm{bytes}$ \\
\hline Number of Packets & $10 / \mathrm{sec}$ \\
\hline Mobility Pause Time & $50 \mathrm{sec}$ \\
\hline Movement Speed & $5,10,15,20$ and $25 \mathrm{~m} / \mathrm{sec}$ \\
\hline Number of Simulation & 20 \\
\hline
\end{tabular}

\section{RESULTS AND DISCUSSION}

\subsection{Simulation Results of the Proposed Method}

This section has been focused to prove the performance of the cluster based multi-source multicast routing protocol in various environments and also its delivery ratio, control overhead and forwarding efficiency have been compared with ODMRP. The Figure 7 shows the average number of cluster formation for 50,100,150 and 200 nodes with various transmission ranges. It proves while our transmission range is going to large the average number of cluster is going to low. Moreover, while number of nodes is maximum the number of cluster also maximum. he Figure 8,9 and 10 proves while the mobile nodes, simulation time and mobility speed are maximum the average number of clusters is also being as maximum. The Figure 11 drawn to prove the delivery ratio in various area sizes. It proves if we increase the area size the delivery ratio is going to low. The Figure 12 explains the delivery ratio with that mobility speed. It proves if the mobility speed is going to maximum the delivery ratio is going to minimum.

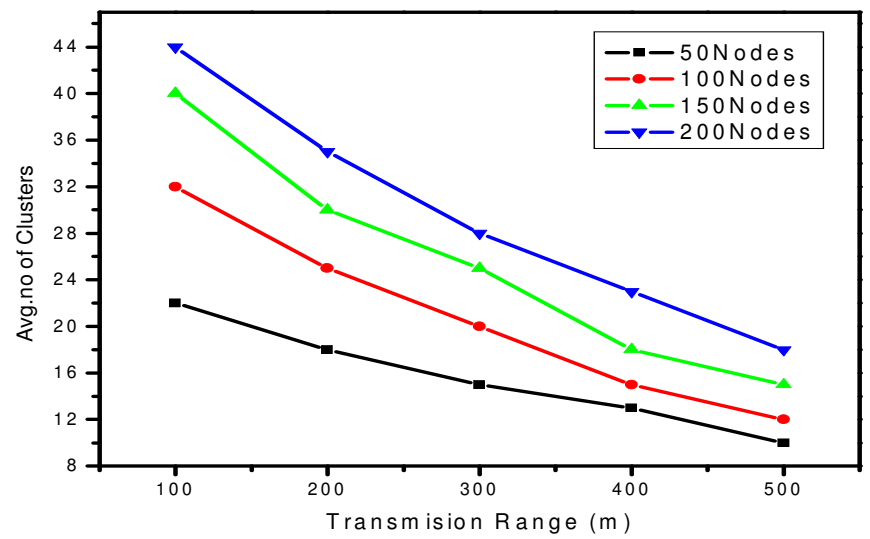

Figure 7. Transmission Range Vs Average Number of Clusters 
International Journal of Computer Networks \& Communications (IJCNC) Vol.3, No.1, January 2011

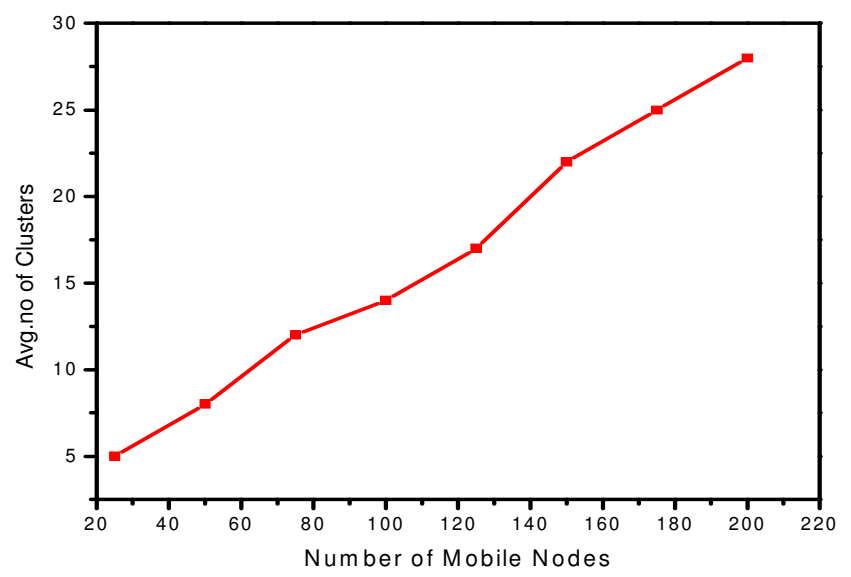

Figure 8. Number of mobile nodes Vs Average Number of Clusters

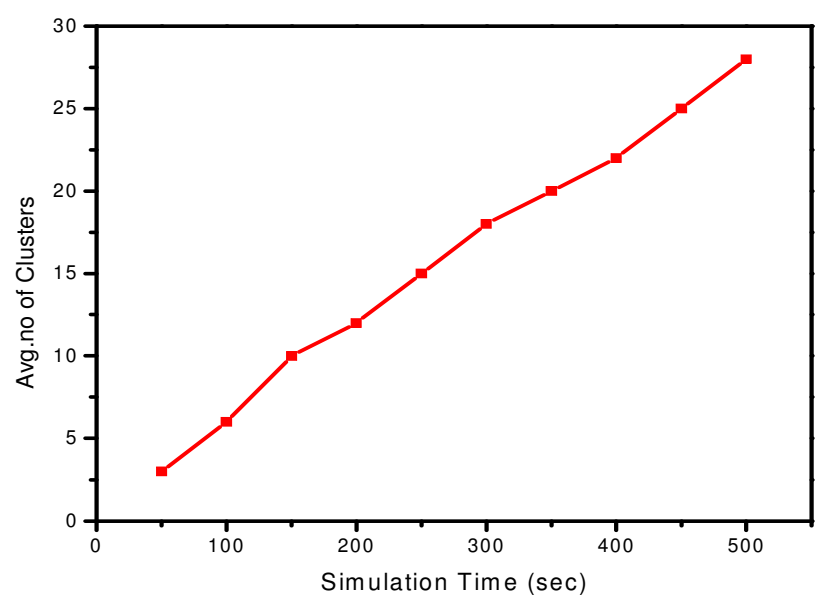

Figure 9. Simulation time Vs Average Number of Clusters

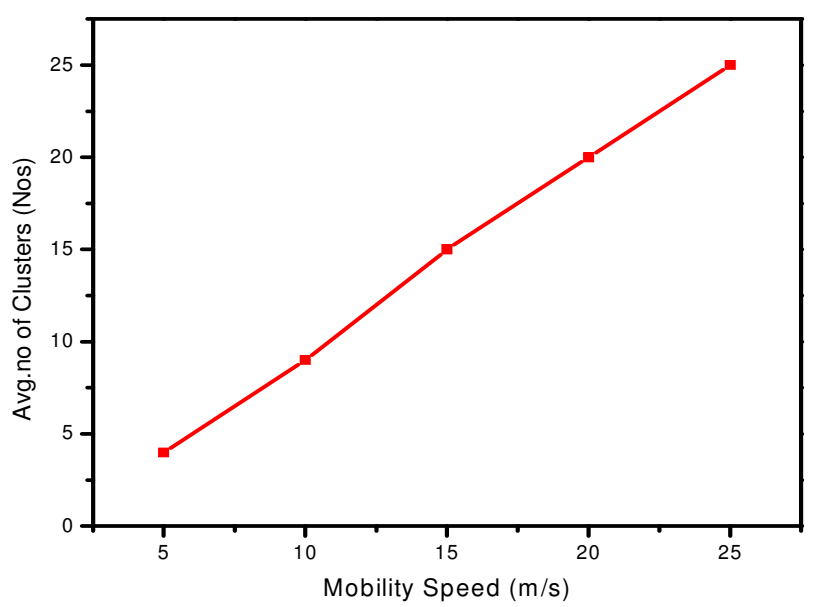

Figure 10. Mobility speed Vs Average Number of Clusters 
International Journal of Computer Networks \& Communications (IJCNC) Vol.3, No.1, January 2011

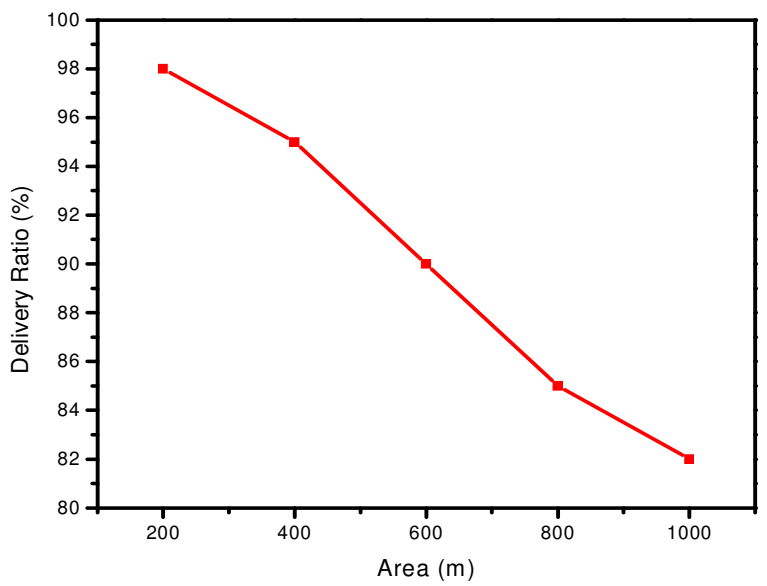

Figure 11. Area Vs Delivery Ratio

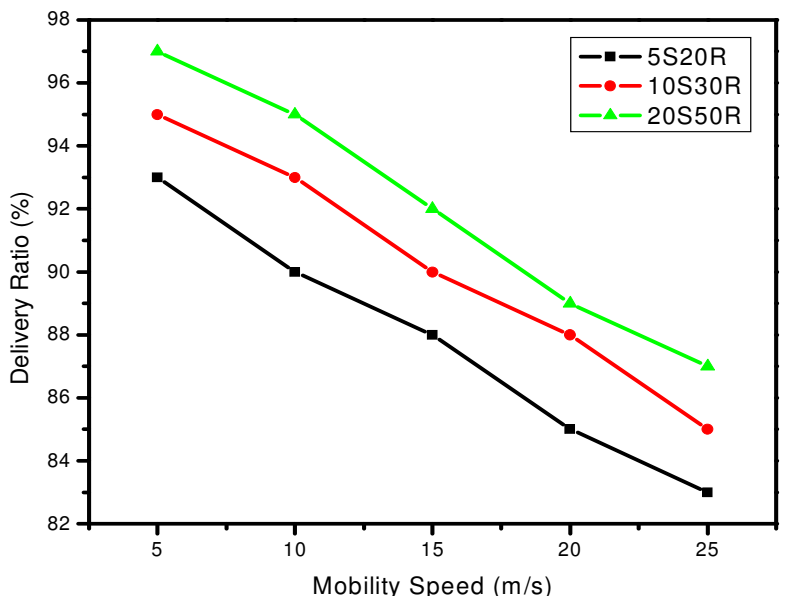

Figure 12. Mobility speed Vs Delivery Ratio

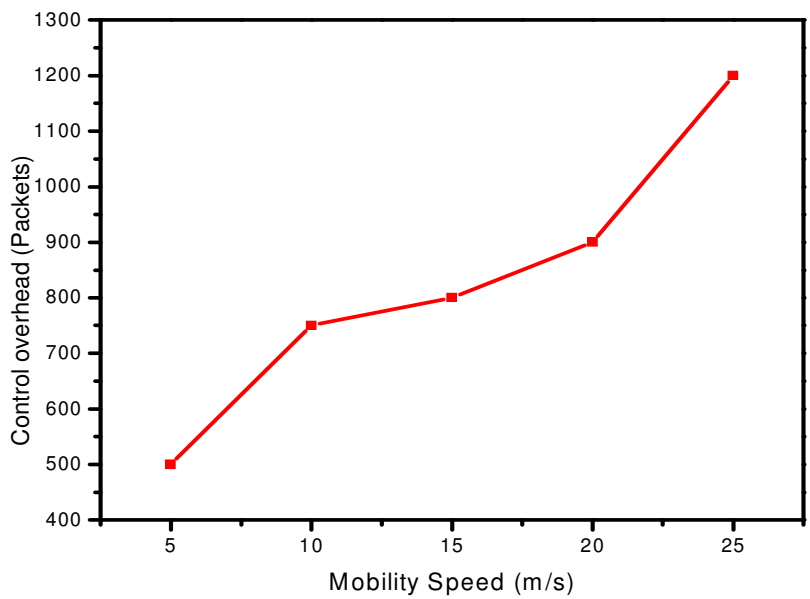

Figure 13. Mobility speed Vs Control overhead 


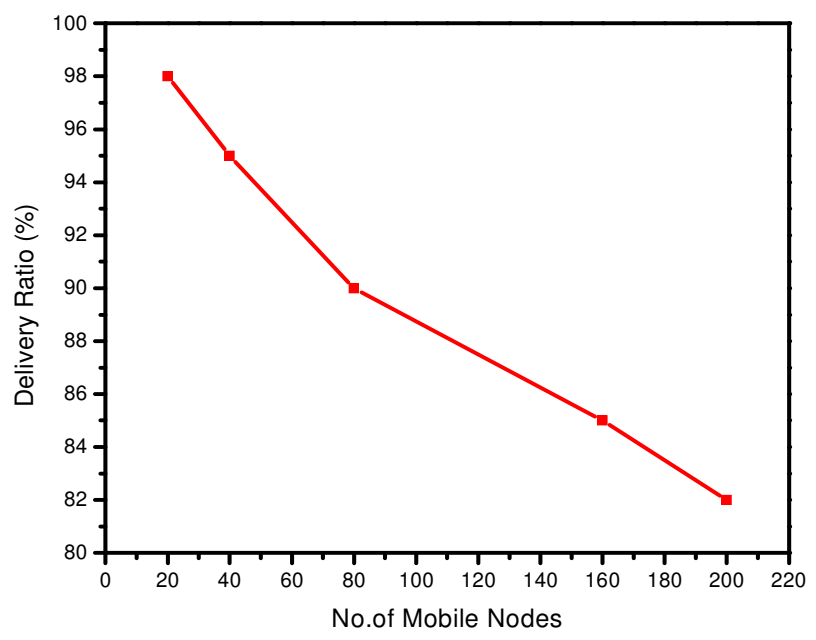

Figure 14.Number of mobile node Vs Delivery Ratio

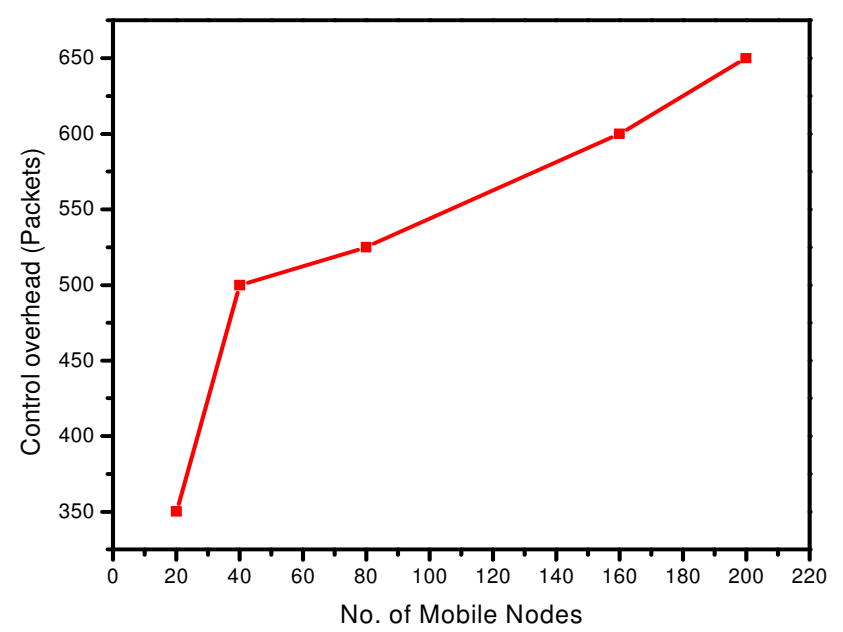

Figure 15.Numbers of mobile nodes Vs Control Overhead

The Figure 13 proves the relationship of mobility speed and control overhead, where the mobility speed is going to maximum the control overhead also going to maximum. The Figure 14 proves, while number of mobile nodes is going to maximum the delivery ratio is going to minimum. The Figure 15 proves, while the number of mobile nodes is going to maximum the control overhead is also going to maximum.

\subsection{Comparison with MAODV and ODMRP}

The packet delivery ratio defined as the number of data packets delivered to multicast receivers versus number of data packets transmitted from multicast senders. Control overhead is the summation of the control messages generated by the protocol over the entire network. In ODMRP, control overhead includes join query and join reply issued by sources and receivers. In CBMRP, control messages for clustering, constructing the multicast route, and maintaining the cluster structure are counted for the control overhead. The forwarding efficiency calculated as number of data packets received by the receivers and number of data packets transmitted by nodes. For the Figure 16, 17 and 18 Source nodes are taken as 1, 5, 10, 15 and 20 and the receiver nodes are taken as 10,20,30,40 and 50 respectively. 


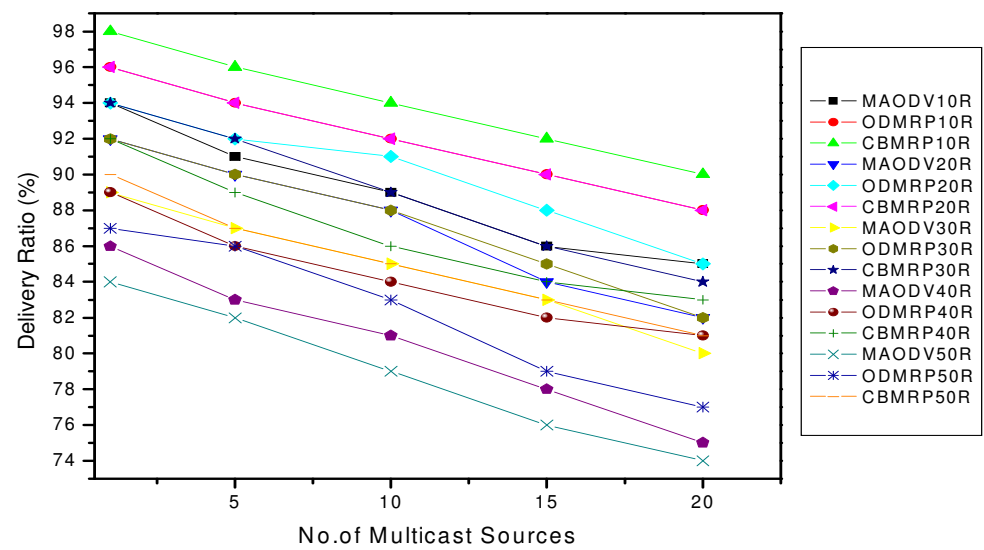

Figure 16. Number of multicast sources Vs Packet Delivery Ratio

The Figure 16 drawn to prove the delivery ratio of CBMRP with existing MAODV and ODMRP routing protocols on increase the number of senders and receivers. It explains while the number of sources and receivers are small, all are giving high packet delivery ratio. If we increase the sources and receivers, the delivery ratio is going to low in all routing protocols. Even though, the CBMRP gives better delivery ratio than other planned routing protocols.
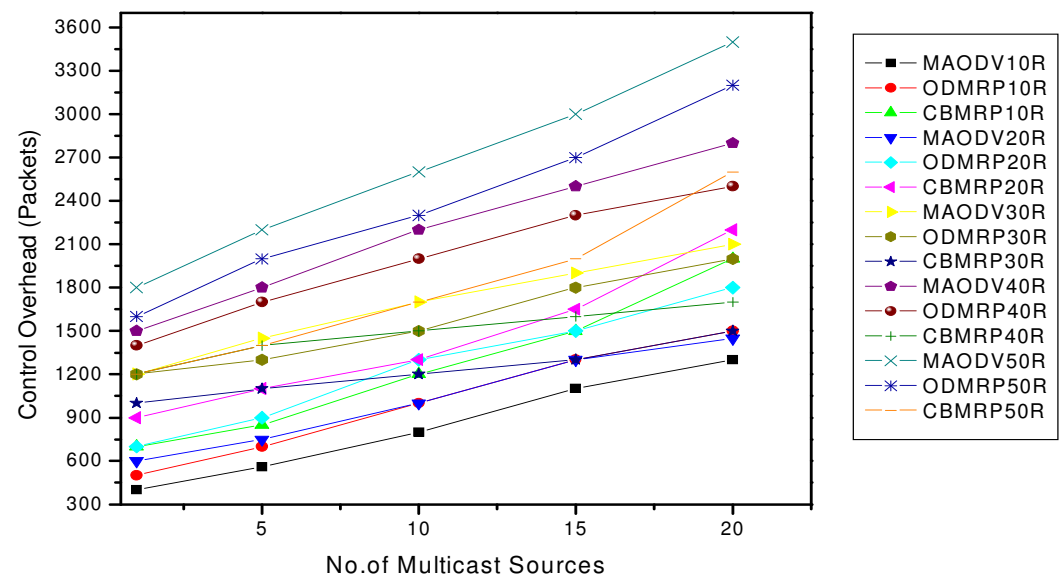

Figure 17. Number of multicast sources Vs Control Overhead

The Figure 17 shows the control overhead of planned routing protocols, which explains when the number of senders and receivers are small the control messages are going as MAODV $<$ ODMRP $<$ CBMRP. But, when the senders and receivers are increasing all are generating the control messages by the MAODV $>$ ODMRP $>$ CBMRP. The MAODV and ODMRP are having small variation overhead than CBMRP. 

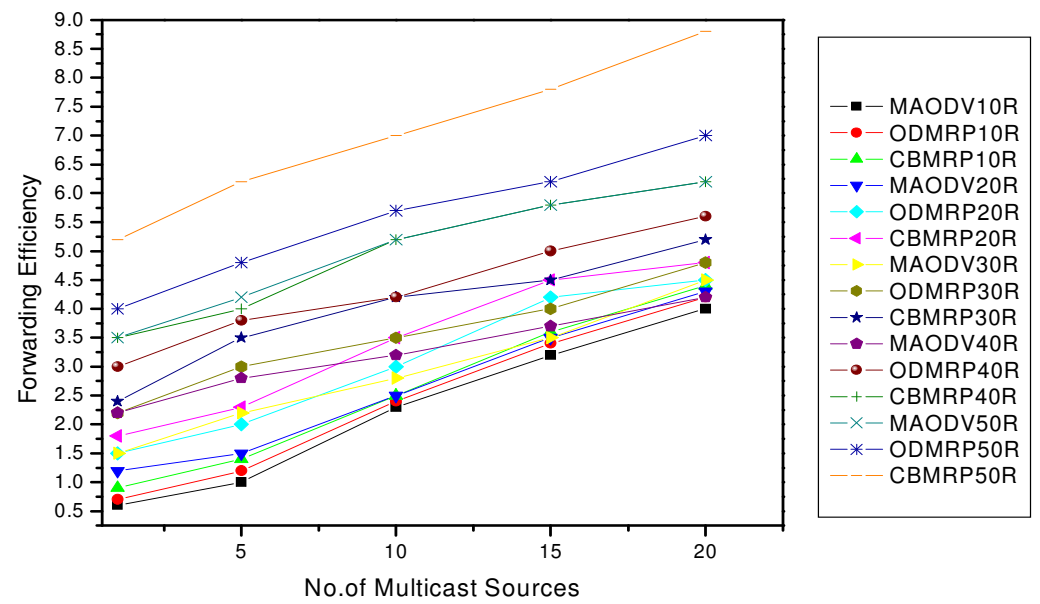

Figure 18. Number of multicast sources Vs Forwarding Efficiency

The Figure 18 drawn for forwarding efficiency of MAODV, ODMRP and CBMRP routing protocols which explains when the number of senders and receivers are small all are having minimum forwarding efficiency with small variation. If we raise the number of sources and receivers, the CBMRP achieves better efficiency due to the cluster structure than MAODV and ODMRP.

\section{CONClusion}

In this paper we analyzed the proposed cluster based multi-source multicast routing protocol performances in mobile ad hoc networks. Due to the cluster structure and setting forwarding clusters, the proposed CBMRP is efficient and robust for multi-source multicasting. Based on this more than one multicast source we can prevent the flooding of route request messages gaining by the individual source. This proposed routing protocol various performance results are discussed in Section 5.1 and in the Section 5.2 this CBMRP deliver ratio, control overhead and forwarding efficiency results are compared with existing MAODV and ODMRP. These results are proving the proposed CBMRP outperforms than MAODV and ODMRP in delivery ratio and forwarding efficiency for mobile ad hoc networks.

\section{REFERENCES}

[1] R. Manoharan and E. Ilavarasan, "Impact of the mobility on the performance of multicast routing protocols in MANET", International Journal of wireless \& mobile networks (IJWMN), vol.2 no.2, 2010, pp 110-119.

[2] Ratish Agarwal and Mahesh Motwani, "Survey of clustering algorithms for MANET", International Journal on Computer Science and Engineering, vol.1 (2), 2009, pp. 98-104.

[3] Arwa Zabian, Ahmed Ibrahim and Fadi Al-Kalani, "Dynamic Head Cluster Election Algorithm for Clustered Ad-Hoc Networks”, Journal of Computer Science 4(1), 2008, pp. $42-50$.

[4] Luo Junhai, Xue Liu and Ye Danxia, "Research on multicast routing protocols for mobile adhoc networks", Computer Networks 52, 2008, pp. 988-997.

[5] Yi.-Yu Su, Shiow-Fein Hwang and Chyi-Ren Dow, "An Efficient cluster-based routing algorithm in ad hoc networks with unidirectional links", Journal of Information Science and Engineering 24, 2007, pp. 1409 - 1428. 
[6] Law, L. K., Krishnamurthy, S. V., and Faloutsos. M., "Understanding and exploiting the tradeoffs between broadcasting and multicasting in mobile ad hoc networks", Trans. on mobile computing, 2007, pp. $264-279$.

[7] Zeyad M. Alfawaer, GuiWei Hua, and Noraziah Ahmed, “A Novel Multicast Routing Protocol for Mobile Ad Hoc Networks”, American Journal of Applied Sciences 4 (5), 2007, pp. 333-338.

[8] Bai, R., and Singhal, M., "DOA: DSR over AODV routing for mobile ad hoc networks", IEEE Transactions on Mobile Computing, 2006, pp.1403-1416.

[9] Yi-Yu Su, Shiow-Fen Hwang and Chyi-Ren Dow, "An Efficient Multi-Source Multicast Routing Protocol in Mobile Ad Hoc Networks" 11th International Conference on Parallel and Distributed Systems (ICPADS'05), IEEE, 2005.

[10] Jane y. Yu and peter h. J. Chong, "A survey of clustering schemes for mobile ad hoc networks", IEEE communications surveys \& tutorials, vol.7. no.1 2005.

[11] Abolfazle Akbari, Mahdi Soruri and Seyed Vahid Jalali, "Survey of stable clustering for Mobile Ad- hoc Networks", second International Conference on Machine Vision, 2009, pp. 3-7.

[12] Krishna Gorantala, "Routing protocols in Mobile Ad-hoc Networks", Master Thesis in Computer Science, Dept.of Comp.Sci., Umea Univ., Swedan, June 2006.

[13] Y.Y. Su, S.F. Hwang, and C.R. Dow, "An Efficient Cluster-Based Routing Algorithm in Ad Hoc Networks with Unidirectional links," Proceedings of International Computer Symposium (ICS2002), pp. 511- 520, Dec 2002.

[14] C.-K. Toh, “Ad Hoc Mobile Wireless Networks: Protocols and Systems”, Prentice Hall, 2002.

[15] Teerawat Issariyakul and Ekram Hossain, "Introduction to Network Simulator (NS2)", ISBN: 978-0-387-71759-3.

[16] S.Park, "NS-2 Tutorial”, [Online]. Available: http://www.isi.edu/nsnam/ns

\section{Authors}

Pandi Selvam Raman received his B.Sc., Degree from Bharathidasan University in 2005. He also received the M.Sc., and M.Phil., Degrees from Alagappa University in 2007 and 2008 respectively. Since July 2008, he is doing research in Computer Science, Department of Computer Science and Engineering, Alagappa University, Karikudi, Tamil Nadu,India. His research interest includes mobile computing, Data Mining \& Warehousing, Ad hoc wireless networks \& security, data miming and computer algorithms.

Dr. Palanisamy Vellaiyan obtained his B.Sc. degree in Mathematics from Bharathidasan University in 1987. He also received the M.C.A., and Ph.D Degrees from Alagappa University in 1990 and 2005 respectively. After that working as Lecturer in AVVM Sri Pushpam College, Poondi from 1990 to 1995, He joined Alagappa University as Lecturer in 1995. He is currently working as Associate Professor and Head of Department of Computer Science and Engineering. He also received the M.Tech., Degree from Bharathidasan University in 2009. He has

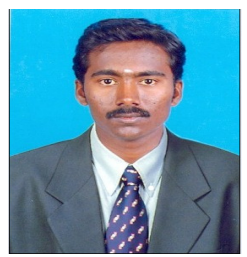
published over 20 journals and conferences and his research interest includes Computer Networks \& Security, Data Mining \& Warehousing, Mobile Communication and computer algorithms. 